Pacific Journal of Mathematics

AMALGAMATING ABELIAN ORDERED GROUPS 


\title{
AMALGAMATING ABELIAN ORDERED GROUPS
}

\author{
KeIth R. Pierce
}

\begin{abstract}
Sums, or amalgamations, of two abelian ordered groups with a subgroup amalgamated are constructed in two ways. These constructions are used to investigate the structure of the class of all amalgamations with the given groups and subgroup fixed, where the class is partially ordered in a natural way. In particular, necessary and sufficient conditions are found for there to be (a) exactly one amalgamation, up to equivalence, and (b) exactly one minimal amalgamation, up to equivalence.
\end{abstract}

It is known [5] that the class of abelian (totally) ordered groups (o-groups) has the amalgamation property. Relying on the injective property of $\eta_{\alpha}$-groups, the indicated proof is existential in nature, and yields no information about the amalgamations. In this paper we present two ways to construct all amalgamations of a subgroup of two abelian o-groups. For the first way we merely consider a certain class of homomorphic images of the abelian amalgamated free product (Theorem 1.2). The technique quickly yields some general information about the structure of the class of all amalgamations of a given subgroup (Corollaries 1.3 and 3.3). The second way is more specific, involving the existence of certain embeddings of the groups into a Hahn group (Theorem 2.8). An amalgamation is called minimal if it admits no o-homomorphisms which are $1-1$ on the component groups. These turn out to be important because every amalgamation is a lexicographic extension of a non-essential group by a minimal amalgamation (Lemma 3.1). In $\S 4$ we use the second construction to determine when there is precisely one minimal amalgamation (Theorem 4.3), and if there is more than one, how many there are (Theorem 4.6). In addition, we determine under what conditions there is exactly one amalgamation (Theorem 4.4).

The possibility exists that these techiques can be adapted to the class of abelian lattice-ordered groups ( $l$-groups) since the author in [6] has shown that the class of abelian $l$-groups has the amalgamation property. The class of $l$-groups, however, does not have this property (see [6, Theorem 3.1]). The author in [6] and N. R. Reilly in [7] have determined some sufficient conditions for amalgamation to occur in this class.

Notation. All groups are additive and abelian. $Z, Q$, and $R$ denote respectively the o-groups of integers, rational numbers, and 
real numbers. $|x|$ stands for the cardinality of $x$, and we let $|R|=c$. We sometimes denote a partially ordered group by $[H, P]$ where $P=H^{+}$is the positive cone (partial order) of $H$. The lexicographic direct sum of the o-groups $G$ and $H$ is written as $G \oplus^{*} H$ (i.e., $0<g+h$ if $0<h$, or $h=0$ and $0<g$ ). The classes of groups and $o$-groups are denoted respectively by $\mathscr{G}$ and $\mathcal{O}$.

The reader should consult [3] for basic facts about o-groups. [4] is also a good reference.

1. Preliminaries; the free product construction. A class $\mathscr{K}$ of similar algebraic structures is said to have the amalgamation property if whenever $G, H$, and $K$ are in $\mathscr{C}$ and $\sigma_{1}: G \rightarrow H$ and $\sigma_{2}: G \rightarrow K$ are embeddings, there exist $L \in \mathscr{K}$ and embeddings $\eta_{1}: H \rightarrow L$ and $\eta_{2}: K \rightarrow L$ such that $\sigma_{1} \eta_{1}=\sigma_{2} \eta_{2}$. If $L$ is generated by $H \eta_{1} \cup K \eta_{2}$ then the triple $\left(L, \eta_{1}, \eta_{2}\right)$ is called an amalgamation of $G$ in $H$ and $K$. For simplification, $\sigma_{1}$ and $\sigma_{2}$ will always be incusion maps, and we may just use $L$ in place of $\left(L, \eta_{1}, \eta_{2}\right)$. For fixed $G, H$, and $K$, we say that $\left(L, \eta_{1}, \eta_{2}\right)$ is freer than $\left(M, \mu_{1}, \mu_{2}\right)$ (denoted $L>M$ ) if there is a homomorphism $\theta: L \rightarrow M$ such that $\eta_{i} \theta=\mu_{i}(i=1,2)$. If $\theta$ is an isomorphism then $L$ and $M$ are equivalent (denoted $L \approx M$ ). One easily shows that $\approx$ is an equivalence relation, $>$ is reflexive and transitive, and $L \approx M$ if and only if $L \succ M$ and $M>L$. $\mathscr{L}_{\mathscr{K}}$ $(G, H, K)$ is a representing set of amalgamations of $G$ in $H$ and $K$ if it consists of exactly one amalgamation out of every equivalence class. $\mathscr{L}_{\mathscr{K}}(G, H, K)$ is partially ordered by $\succ$, and any two such representing sets are canonically isomorphic. Our object is to determine much of the structure of $\mathscr{L}_{0}(G, H, K)$.

If $\mathscr{L}_{\mathscr{S}}(G, H, K)$ has a greatest element, it is the free product in $\mathscr{K}$ of $H$ and $K$ with $G$ amalgamated. Of interest here is the free product $\left(F, \mu_{1}, \mu_{2}\right)$ in $\mathscr{G}$. It can be represented as

$$
F=(H \oplus K) / G^{*}
$$

where $\mu_{1}$ and $\mu_{2}$ are the natural embeddings and $G^{*}$ consists of all pairs $\left(g \mu_{1},-g \mu_{2}\right)(g \in G)$.

Let $X^{\prime}$ denote the divisible closure of the o-group $X$. The order on $X$ can be extended to $X^{\prime}$ by letting $x^{\prime}$ be positive if $n x^{\prime} \in X^{+}$for some positive integer $n$. Any $o$-homomorphism $\eta: X \rightarrow Y$ has a unique extension to $\eta^{\prime}: X^{\prime} \rightarrow Y^{\prime}$, and $\eta^{\prime}$ is $1-1$ if and only if $\eta$ is $1-1$. The next lemma justifies restricting our attention to the class of divisible o-groups. The proof is straightforward.

Lemma 1.1. If $\left(L, \eta_{1}, \eta_{2}\right)$ is an amalgamation of $G$ in the ogroups $H$ and $K$, then $\left(L^{\prime}, \eta_{1}^{\prime}, \eta_{2}^{\prime}\right)$ is an amalgamation of $G^{\prime}$ in $H^{\prime}$ 
and $K^{\prime}$, and the induced map $L \rightarrow L^{\prime}$ is an order isomorphism from $\mathscr{L}_{0}(G, H, K)$ onto $\mathscr{L}_{0}\left(G^{\prime}, H^{\prime}, K^{\prime}\right)$.

From now on we will consider only divisible groups and subgroups. We will make frequent use of the fact that divisible subgroups are direct summands. Since division in an o-group is unique, we can alternately consider them as rational vector spaces and subspaces.

Let $V$ be a rational vector space. Define the set $O Q(V)$ of orderable quotients of $V$ to be the collection of all o-groups of the form $[V / I, P]$ where $I$ is a rational subspace of $V(V / I$ is torsionfree, hence orderable). Partially order $O Q(V)$ by defining $[V / I, P] \geqq$ $[V / J, Q]$ if and only if $I \subseteq J$ and the natural homomorphism

$$
v+I \longmapsto v+J
$$

preserves order.

THEOREM 1.2. Let $G$ be a subgroup of each of the o-groups $H$ and $K$ and let $\left(F, \mu_{1}, \mu_{2}\right)$ be the free product in $\mathscr{G}$ of $H$ and $K$ with $G$ amalgamated. Then $\mathscr{L}_{0}(G, H, K)$ is isomorphic to the subset $\mathscr{F}$ of $O Q(F)$ consisting of all $[F / I, P]$ such that

(1) $I \cap\left(H \mu_{1} \cup K \mu_{2}\right)=0$, and

(2) $H^{+} \mu_{1}+K^{+} \mu_{2}+I \subseteq P$.

Proof. If $[F / I, P]$ is in $\mathscr{F}$ and $\phi(I): F \rightarrow F / I$ is natural then $\mu_{1} \phi(I)$ and $\mu_{2} \phi(I)$ are $o$-embeddings. Thus the collection of $([F / I, P]$, $\left.\mu_{1} \phi(I), \mu_{2} \phi(I)\right),[F / I, P] \in \mathscr{F}$, is a collection of amalgamations in $\mathcal{O}$ of $G$ in $H$ and $K$. Furthermore, one easily checks that the partial order on $\mathscr{F}$ is identical with the induced partial order $\succ$. It remains to show that every equivalence class of amalgamations is represented. If $\left(L, \eta_{1}, \eta_{2}\right) \in \mathscr{L}_{0}(G, H, K)$ then $L$ is also an amalgamation in $\mathscr{G}$, whence there is a group epimorphism $\phi: F \rightarrow L$ such that $\mu_{i} \phi=\eta_{i}(i=1,2)$. If $I=\operatorname{ker}(\phi)$ and $\theta: F / I \rightarrow L$ is natural then evidently $\left(L, \eta_{1}, \eta_{2}\right)$ and $\left(\left[F / I, L^{+} \theta^{-1}\right], \mu_{1} \phi, \mu_{2} \phi\right)$ are equivalent. This completes the proof.

Corollary 1.3. Let $\mathscr{L}=\mathscr{L}_{0}(G, H, K)$. Then

(1) $\mathscr{L}$ is an inverse root system; i.e., the set of all elements exceeded by a given element forms a chain,

(2) Every element of $\mathscr{L}$ exceeds exactly ione minimal element, and is exceeded by at least one maximal element, and

(3) Each component of $\mathscr{L}$ (i.e., the set of elements exceeding a minimal element) is a lower semilattice. 
Proof. The set of ordered quotients below $[F / I, P]$ in $\mathscr{F}$ is evidently antiisomorphic to the set of all subgroups $J, I \leqq J \subseteq F, J / I$ convex in $[F / I, P]$, ordered by inclusion. But this is a totally ordered set. Hence (1) is proven. For $[F / I, P] \in \mathscr{F}$ let $Q^{\prime}$ be a total order on $I$ and let $Q$ be the total order $Q^{\prime} \cup\{x \in F: x+I \in P\}$ on $F$. Then $[F, Q]$ is maximal in $\mathscr{F}$ and exceeds $[F / I, P]$. Going the other way let $J$ be the largest subgroup of $F$ such that $I \subseteq J, J / I$ is convex in $[F / I, P]$ and disjoint from $\left(H \mu_{1}+K \mu_{2}+I\right) / I$. If $Q$ is the order on $F / J$ induced by $P$, then $[F / J, Q]$ is minimal and exceeded by $[F / I, P]$. By (1) it is unique. Thus (2) is proven. Finally suppose $[F / I, P]$ and $\left[F / I^{\prime}, P^{\prime}\right]$ both exceed the minimal element $\left[F / J^{\prime}, Q^{\prime}\right]$ of $\mathscr{F}$. Then the collection of subgroups $J$ such that $J / I$ is convex in $[F / I, P]$ and $J / I^{\prime}$ is convex in $\left[F / I^{\prime}, P^{\prime}\right]$ is nonempty and linearly ordered by inclusion. If $J^{*}$ is their intersection, then evidently $\left[F / J^{*}, P+J^{*} / J^{*}\right]$ is in $\mathscr{F}$ and the infimum of the two given ordered quotients. This proves (3) and completes the proof of the corollary.

\section{Constructing amalgamations using Hahn embeddings.}

LEMma 2.1 [7]. Every partial order on a set can be extended to a total order.

LEMmA 2.2. The class of ordered sets has the amalgamation property. More specifically, if $A$ is a subset of the ordered sets $B$ and $\Gamma$, and $\beta^{*}$ and $\gamma^{*}$ determine the same cut of $A-i . e ., \alpha \leqq \beta^{*}$ if and only if $\alpha \leqq \gamma^{*}(\alpha \in A)$-then there is an amalgamation $\Delta\left(\Delta^{\prime}\right)$ of $A$ in $B$ and $\Gamma$ in which $\beta^{*}<\gamma^{*}\left(\beta^{*}=\gamma^{*}\right)$.

Proof. Let $B \cap \Gamma=A$ and extend the orders on $B$ and $\Gamma$ to a partial order of $B \cup \Gamma$ by letting $\beta \leqq \gamma$ if $\beta \leqq \alpha \leqq \gamma$ for some $\alpha \in A$ or if $\beta, \beta^{*}, \gamma$, and $\gamma^{*}$ all determine the same cut of $A$, and letting. $\gamma \leqq \beta$ if $\gamma \leqq \alpha \leqq \beta$ for some $\alpha \in A$. Its extension to a total order yields $\Delta$ as desired. For $\Delta^{\prime}$, first identify $\beta^{*}$ and $\gamma^{*}$, then proceed as above.

LEMMA 2.3. The class of archimedian o-groups has the amalgamation property. If the amalgamated subgroup is nonzero, then any two amalgamations are equivalent.

Proof. Let $G$ be a subgroup of each of the archimedian ogroups $H$ and $K$, and let $\nu_{1} ; H \rightarrow R$ and $\eta_{2}: K \rightarrow R$ be o-embeddings. Since every o-isomorphism between subgroups of $R$ is realized as multiplication by a positive real number, there exists $0<r \in R$ such 
that $\left(g \nu_{1}\right) r=g \eta_{1}$ for all $g \in G$. Putting $\eta_{1}=\nu_{1} r, L=H \eta_{1}+K \eta_{2}$, we have the desired amalgamation $\left(L, \eta_{1}, \eta_{2}\right)$. If $\left(M, \mu_{1}, \mu_{2}\right)$ is also an amalgamation with $M \subseteq R$, then $\eta_{1}^{-1} \mu_{1}=s$ and $\eta_{2}^{-1} \mu_{2}=t$ for some $0<s, t \in R$. If $G \neq 0$ we must have $s=t$, and thus $L$ and $M$ are equivalent via the o-isomorphism induced by $s$.

The following concept is introduced for notational convenience. $A \Gamma$-valuation of an o-group $H$ is a map $\gamma \mapsto\left(H^{r}, H_{\gamma}\right)$ from the ordered set $\Gamma$ to pairs of convex subgroups of $H$ satisfying

(1) $H_{r} \subseteq H^{r}$. If $H_{r} \subset H^{\gamma}$, then $H^{\gamma}$ covers $H_{r}$,

(2) $\gamma \leqq \delta$ implies $H^{r} \leqq H_{\delta}$, and

(3) If $h \neq 0$ then $h \in H^{\gamma} \backslash H_{\gamma}$ for some $\gamma \in \Gamma$.

$H$ is called a $\Gamma$-group. $\gamma$ is a value of $h$ if $h \in H^{r} \backslash H_{\gamma}$. The spine of $H$ is the subset $\Gamma_{a}=\left\{\gamma: H_{r} \subset H^{r}\right\}$. The valuation is proper if $\Gamma=\Gamma_{a}$. Evidently all proper valuations are identical. For basic results on $\Gamma$-valuations see Conrad [2].

An easy consequence of the definition is the

LeMma 2.4. For every $\gamma \in \Gamma$,

$$
H^{\gamma}=\bigcap H_{o}\left(\gamma<\delta, \delta \subset \Gamma_{a}\right) .
$$

A $\Delta$-valuation on $H$ is said to extend a $\Gamma$-valuation if $\Gamma \subseteq \Delta$ and $\left(H^{\delta}, H_{\delta}\right)=\left(H^{\gamma}, H_{\gamma}\right)$ whenever $\delta=\gamma$ and $\gamma \in \Gamma$.

Lemma 2.5. If $\Gamma \leqq \Delta$ then every $\Gamma$-valuation on $H$ has a unique extension to a $\Delta$-valuation.

Proof. Evidently defining

$$
H^{\delta}=H_{\delta}=\bigcap H_{r}\left(\delta<\gamma, \gamma \in \Gamma_{a}\right)
$$

for all $\delta \in \Delta \backslash \Gamma$ produces an extension. To prove uniqueness we note that the spine of any extension is $\Gamma_{a}$, so by Lemma 2.4 all extensions are equal to the one just defined.

An $o$-embedding $\sigma: G \rightarrow H$ between $\Gamma$-groups is a $\Gamma$-embedding if $G_{r} \sigma=H_{r} \cap G \sigma$ and $G^{r} \sigma=H^{r} \cap G \sigma . \quad G$ is a $\Gamma$-subgroup of $H$ if the inclusion map is a $\Gamma$-embedding. Every subgroup of a $\Gamma$-group admits a unique $\Gamma$-valuation making it a $\Gamma$-subgroup.

For each $\gamma \in \Gamma$ let $R_{\gamma}$ be an archimedian o-group. The Hahn group $V\left(\Gamma, R_{\gamma}\right)$ is the subgroup of $\Pi R_{r}(\gamma \in \Gamma)$ consisting of all functions with inversely well-ordered support, ordered by letting $f$ be positive if its greatest nonzero component is positive. $V\left(\Gamma, R_{r}\right)$ admits a natural $\Gamma$-valuation where $V^{\gamma}\left(V_{\gamma}\right)=\{f ; f(\delta)=0$ for all $\delta>\gamma(\delta \geqq \gamma)\}$. If $H$ is a $\Gamma$-group we define $V(H)=V\left(\Gamma, H^{r} / H_{r}\right)$. Banaschewski's proof [1] that a divisible $\Gamma$-group $H$ is $\Gamma$-embeddable in $V(H)$, although 
elegant, is too restrictive ( $\S 4)$, so we will use Conrad's decomposition proof from [2], outlined below. A collection $T$ of subgroups $T_{r}$ of a $\Gamma$-group $H$ is a $\Gamma$-decomposition of $H$ if

(1) $H_{r}=H^{r} \cap T_{r}$,

(2) $H=H^{\gamma}+T_{r}$, and

(3) For every $h, h \in T_{r}$ for all but at inversely well-ordered subset of $\Gamma$.

The map $\bar{T}: H \rightarrow V(H), h \bar{T}(\gamma)=\left(h+T_{\gamma}\right) \cap H^{\gamma}$, is a $\Gamma$-embedding. If $S$ is a $\Gamma$-decomposition of the $\Gamma$-subgroup $G$, then there is a $\Gamma$ decomposition $T$ of $H$ such that $S=T \cap G$ (i.e., $S_{r}=T_{r} \cap G$ for all $\gamma \in \Gamma)$.

A natural embedding $\sigma: V\left(\Gamma, P_{r}\right) \rightarrow V\left(\Gamma, R_{r}\right)$ is one which is induced by o-embeddings $\sigma_{\gamma}: P_{\gamma} \rightarrow R_{\gamma}$, where $f \sigma(\gamma)=f(\gamma) \sigma_{\gamma}$. In particular, if $G$ is a $\Gamma$-subgroup of $H$, then the natural o-embeddings

$$
\sigma_{r}: G^{r} / G_{r} \longrightarrow H^{r} / H_{r}
$$

induce $\sigma: V(G) \rightarrow V(H)$. One more fact from [2]: if $S$ and $T$ are $\Gamma$-decomposition on $G$ and $H$ respectively and $S=T \cap G$, then $\bar{S} \sigma=$ $\bar{T} \mid G$.

The standard amalgamation. For the remainder of the paper let $G$ be an ordered subgroup of the o-groups $H$ and $K$, and let

$$
\alpha \longmapsto\left(G^{\alpha}, G_{\alpha}\right)(\alpha \in A), \beta \longmapsto\left(H^{\beta}, H_{\beta}\right)(\beta \in B),
$$

and $\gamma \rightarrow\left(K^{r}, K_{r}\right)(\gamma \in \Gamma)$ be proper valuations of the respective groups. There are unique embeddings of $A$ in $B$ and $\Gamma$ such that $G^{\alpha}=H^{\alpha} \cap G=K^{\alpha} \cap G$ and $G_{\alpha}=H_{\alpha} \cap G=K_{\alpha} \cap G$. By Lemma 2.2 let $\Delta$ be an amalgamation of $A$ in $B$ and $\Gamma$. By Lemma 2.5 extend to $\Delta$-valuations on $H$ and $K$. Evidently the maps

$$
\delta \longmapsto\left(H^{\delta} \cap G, H_{\delta} \cap G\right)
$$

and

$$
\delta \longmapsto\left(K^{\delta} \cap G, K_{\delta} \cap G\right)
$$

are $\Delta$-valuations on $G$ which extend the given $A$-valuation. Thus by 2.5 they are the same, and we can therefore consider $G$ as a $\Delta$-subgroup of each of $H$ and $K$. Let $S, T$, and $U$ be $\Delta$-decompositions of $G, H$, and $K$ respectively such that $S=T \cap G=U \cap G$, and let $\sigma_{1}: H \rightarrow V(H)$ and $\sigma_{2}: K \rightarrow V(K)$ be natural $\Delta$-embeddings. Finally, by 2.3 let $\left(R_{\delta}, \nu_{1 \delta}, \nu_{2 \delta}\right)$ be an archimedian amalgamation of $G^{\delta} / G_{\delta}$ in $H^{\delta} / H_{\delta}$ and $K^{\delta} / K_{\delta}$, and let $\nu_{1}: V(H) \rightarrow V\left(\Delta, R_{\delta}\right)$ and $\nu_{2}: V(K) \rightarrow V\left(\Delta, R_{\delta}\right)$ be natural $\Delta$-embeddings. $\bar{T} \nu_{1}$ and $\bar{U} \nu_{1}$ agree on $G$, whence $\left(H \bar{T} \nu_{1}=\right.$ $\left.K \bar{U} \nu_{2}, \bar{T} \nu_{1}, \bar{U}_{2}\right)$ is an amalgamation of $G$ in $H$ and $K$. We will call it a standard amalgamation. 
Evidently the spine of a standard amalgamation is the amalgamation $\Delta$ of the spines. Thus every standard amalgamation is minimal. The converse is false, as this example shows:

ExAmple 2.6. Let $H=K=R \oplus^{*} R$ and let $G$ be all elements with second component zero. Embed $H$ and $K$ in $R \oplus^{*} R \oplus^{*} R$ by letting

$$
(x, y) \eta_{1}=(x, o, y)
$$

and

$$
(x, y) \eta_{2}=(x, y, y) \text {. }
$$

The induced amalgamation is not standard since its spine properly contains an amalgamation of the spine of $G$ in those of $H$ and $K$. If $\pi$ is the projection on the middle factor then replacing $\eta_{2}$ by $\eta_{2}-\eta_{2} \pi$ yields a standard amalgamation. This motivates the following construction.

Let $\left(L, \eta_{1}, \eta_{2}\right)$ be a minimal amalgamation and suppose that $L$ is an 0 -subgroup of the o-group $N$. If $\pi: H \rightarrow N$ is a group homomorphism satisfying

(1) $G \leqq \operatorname{ker}(\pi)$, and

(2) $|h \pi| \ll\left|h \eta_{1}\right|$ for all $h \in H$ (that is $\left|h \eta_{1}\right|$ exceeds every multiple of $|h \pi|)$, then we can form an amalgamation $\left(M, \mu_{1}, \mu_{2}\right)$ by defining $\mu_{1}=\eta_{1}+\pi, \mu_{2}=\eta_{2}$, and $M=H \mu_{1}+K \mu_{2}$. We call $M$ an expansion of $L$.

A $\Delta$-subgroup $H$ of $L$ is a c-subgroup if each of the natural embeddings $H^{\delta} / H_{\delta} \rightarrow L^{\delta} / L_{\delta}$ is surjective.

LEMMA 2.7 [2]. If $H$ is a c-subgroup of $L$ and $T$ is a 4 -decomposition of $L$ then $T \cap H$ is a $\Delta$-decomposition of $H$.

REMARK. This lemma, and hence also the proof of the following theorem, fails if we use Banaschewski functions instead of decompositions. For example, let $\Gamma$ be the set of strictly negative integers, $L=V\left(\Gamma, R_{\delta}\right)\left(R_{\delta}=R\right)$ and let $H$ be the divisible subgroup generated $\Sigma R_{\delta}(\gamma \leqq-2)$ and the element $(\cdots, 1,1,1)$. If $\pi_{L}$ is the natural Banaschewski function on $L$, then $H \cap L^{-2} \pi_{L}=\phi$, and hence $\pi_{L}$ is not the extension of any Banaschewski function on $H$.

THEOREM 2.8. Every amalgamation is equivalent to an expansion of a standard amalgamation.

Proof. Suppose that $\left(M, \mu_{1}, \mu_{2}\right)$ is an amalgamation and $\Delta^{\prime}$ is its spine. When $B$ and $\Gamma$ are naturally embedded in $\Delta^{\prime}$, the embeddings agree on $A$, so some amalgamation $\Delta$ of $A$ in $B$ and $\Gamma$ is embedded 
in $\Delta^{\prime}$. We consider $G, H$, and $K$ as $\Delta^{\prime}$-groups and $\mu_{1}$ and $\mu_{2}$ as $\Delta^{\prime}$ embeddings. Let $S$ and $U$ be $\Delta^{\prime}$-decompositions of $G$ and $K$ respectively such that $S=U \cap G$. By choosing an appropriate $\Delta^{\prime}$-decomposition of $M$ we can assume that $\mathrm{M}$ is a $\Delta^{\prime}$-subgroup of $V(M)$, and if $T^{\prime}$ is the natural $\Delta^{\prime}$-decomposition of $V(M)$, then

$$
U=T^{\prime} \cap K \text { (i.e., } U_{\delta}=\left(T_{\delta}^{\prime} \cap K \mu_{2}\right) \mu_{2}^{-1} \text { : }
$$

we will abuse the notation similarly throughout the proof). Let $\nu_{1_{\delta}}$ and $\nu_{2_{\delta}}$ be the natural embeddings of $H^{\delta} / H_{\delta}$ and $K^{\delta} / K_{\delta}$ respectively into $M^{\delta} / M_{\tilde{\delta}}$, inducing $\nu_{1} ; V(H) \rightarrow V(M)$ and $\nu_{2}: V(K) \rightarrow V(M)$. Note that $\nu_{1_{\delta}}$ and $\nu_{\nu_{\delta}}$ agree on $G^{\delta} / G_{\delta}$ and the choice of decompositions implies $\mu_{2}=\bar{U} \nu_{2}$. Let $\rho_{\delta}$ be a projection of $M^{\delta} / M_{\delta}$ upon $\left(H^{\delta} / H_{\delta}\right) \nu_{1_{\delta}}$ and define $\eta_{1}: H \rightarrow V(M)$ by $h \eta_{1}(\delta)=h \mu_{1}(\delta) \rho_{\delta}$. If $h \in H^{\delta} \backslash H_{\delta}$ then $h \mu_{1}(\delta)=\left(h+H_{\delta}\right) \nu_{1 \delta}$, $h \eta_{1}(\delta)=h \mu_{1}(\delta)$, and $h \mu_{1}\left(\delta^{\prime}\right)=h \eta_{1}\left(\delta^{\prime}\right)=0$ for all $\delta^{\prime}>\delta$. It follows that $\eta_{1}$ is an $o$-embedding. By the choice of decompositions again,

$$
g \mu_{1}(\delta) \in\left(H^{\delta} / H_{\delta}\right) \nu_{1 \delta}
$$

for all $\delta \in \Delta^{\prime}$ and all $g \in G$, whence $\mu_{1}$ and $\eta_{1}$ agree on $G$. Therefore, if we put $\eta_{2}=\mu_{2}$ and $L=H \eta_{1}+K \eta_{2}$, then $\left(L, \eta_{1}, \eta_{2}\right)$ is an amalgamation. Furthermore $M$ is an expansion of $L$ by the homomorphism $\pi=\mu_{1}-\eta_{1}$. It remains to show that $L$ is a standard amalgamation. Evidently the spine of $L$ is precisely $\Delta$, so we can pare down to $\Delta$-groups and $\Delta$-decompositions. $T^{\prime \prime}=T^{\prime} \cap V(H)$ is evidently the natural $\Delta$-decomposition of $V(H)$. Since $\eta_{1} \nu_{1}^{-1}$ embeds $H$ as a $c$-subgroup of $V(H)$, then by Lemma 2.7, $T=T^{\prime \prime} \cap H=T^{\prime} \cap H$ is a decomposition of $H$. Now $T \cap G=S$ : since $g \mu_{1}(\delta) \in\left(H^{\delta} / H_{\delta}\right) \nu_{1_{\delta}}$ for all $g \in G$ and all $\delta \in \Delta$, then we have the following chain of equivalences: $g \in T_{\delta} \Leftrightarrow g \eta_{1} \nu_{1}^{-1} \in T_{\delta}^{\prime \prime} \Leftrightarrow g \eta_{1} \in T_{\delta}^{\prime} \Leftrightarrow g \mu_{1} \in T_{o}^{\prime} \Leftrightarrow g \mu_{2} \in T_{\delta}^{\prime} \Leftrightarrow g \in S_{\delta}$. Finally, we show that $\eta_{1}=\bar{T} \nu_{1}$. Let $h \in H, \delta \in \Delta$. There exists $h^{*} \in H^{\delta}$ such that

$$
h \eta_{1}(\delta)=\left(h^{*}+H_{\delta}\right) \nu_{1 \delta}=h^{*} \eta_{1}(\delta) .
$$

Then $\left(h-h^{*}\right) \eta_{1} \in T_{\delta}^{\prime}$, whence $h-h^{*} \in T_{\dot{\delta}}$. Thus

$$
h \eta_{1}(\delta) \nu_{1 \delta}^{-1}=h^{*}+H_{\delta}=\left(h^{*}+T_{\delta}\right) \cap H^{\delta}=\left(h+T_{\delta}\right) \cap H^{\delta}=h \bar{T}(\delta) .
$$

Thus $\eta_{1}=\bar{T} \nu_{1}$, and it follows that

$$
\left(L, \eta_{1}, \eta_{2}\right)=\left(H \bar{T} \nu_{1}+K \bar{U} \nu_{2}, \bar{T} \nu_{1}, \bar{U} \nu_{2}\right)
$$

is standard.

3. The structure of components of $\mathscr{L}$. Let $\left(L, \eta_{1}, \eta_{2}\right)$ be an amalgamation and let 


$$
H_{L}=\left(H \eta_{1} \cap K \eta_{2}\right) \eta_{1}^{-1}
$$

and

$$
K_{L}=\left(H \eta_{1} \cap K \eta_{2}\right) \eta_{2}^{-1}
$$

Evidently there is a unique o-isomorphism $h \mapsto h^{*}$ from $H_{L}$ onto $K_{L}$ such that $h \eta_{1}=h^{*} \eta_{2}$. If $G=H_{L}$ - that is, $H \eta_{1} \cap K \eta_{2}=G \eta_{1}$ - we say that the amalgamation has the strong intersection property.

We form next a particular kind of expansion. Let $H=H_{L} \oplus H_{L}^{\prime}$ and let $\rho$ be the projection of $H$ on $H_{L}$. Let $C$ be an 0 -group, $\pi$ : $H_{L} \rightarrow C$ a group epimorphism such that $G \leqq \operatorname{ker}(\pi)$. Embed $H$ and $K$ in $C \oplus^{*} L$ by defining $\mu_{1}=\eta_{1}+\rho \pi$ and $\mu_{2}=\eta_{2}$. Evidently $\left(H \mu_{1}+K \mu_{2}, \mu_{1}, \mu_{2}\right)$ is an amalgamation which is an expansion of $L$ by the homomorphism $\rho \pi$, and which is freer than $L$ via the natural projection map. We will call such a construction a vertical expansion of $L$.

LEMMA 3.1. Every amalgamation freer than $L$ is equivalent to a vertical expansion of $L$.

Proof. Let $\left(M, \mu_{1}, \mu_{2}\right)>\left(L, \eta_{1}, \eta_{2}\right)$ via $\theta$ and let $C=\operatorname{ker}(\theta)$. Note that $c \in C$ if and only if $c=h \mu_{1}-h^{*} \mu_{2}$ for some $h \in H_{L}$. This implies that $C \cap\left(H_{L}^{\prime} \mu_{1}+K \mu_{2}\right)=0$. Thus $M=C \oplus^{*} D$ for some subgroup $D$ which contains $H_{L}^{\prime} \mu_{1}+K \mu_{2}$. Let $\rho$ project $H$ on $H_{L}$, and $\tau_{1}$ and $\tau_{2}$ project $M$ on $C$ and $D$ recpectively. By the above representation of elements of $C, \tau_{1}$ maps $H_{L} \mu_{1}$ onto $C$. Let $\pi=\rho \mu_{1} \tau_{1}$. Then $\mu_{2}=\mu_{2} \rho$ since $K \mu_{2} \subseteq D$, and

$$
h \mu_{1}=h \rho \mu_{1}+(h-h \rho) \mu_{1}=h \rho \mu_{1} \tau_{1}+h \rho \mu_{1} \tau_{2}+(h-h \rho) \mu_{1}=h \pi+h \mu_{1} \tau_{2} \text {. }
$$

Thus $M$ is a vertical expansion of the amalgamation $\left(D, \mu_{1} \tau_{2}, \mu_{2} \tau_{2}\right)$. But $D$ is equivalent to $L$ via $\tau_{2}^{-1} \theta$.

TheOREM 3.2. Let $\mathscr{M}$ be a component of $\mathscr{L}_{0}(G, H, K)$ with least member $\left(L, \eta_{1}, \eta_{2}\right)$. Then $\mathscr{C}$ is order isomorphic to $O Q\left(H_{L} / G\right)$.

Proof. Let $M \leqq C \oplus^{*} L$ and $M^{\prime} \subseteq C^{\prime} \oplus^{*} L$ be vertical expansions on $L$ induced by $\pi$ and $\pi^{\prime}$ respectively. One easily checks that $M \succ M^{\prime}$ if and only if $\operatorname{ker}(\pi) \subseteq \operatorname{ker}\left(\pi^{\prime}\right)$ and the induced homomorphism from $C$ to $C^{\prime}$ preserves order; that is, $M>M^{\prime}$ if and only if $H_{L} / \operatorname{ker}(\pi) \geqq H_{L} / \operatorname{ker}\left(\pi^{\prime}\right)$ in $O Q\left(H_{L} / G\right)$. This provides the desired isomorphism.

COROLlaRY 3.3. Let $\delta$ be the dimension of $H_{L} / G$ as a rational vector space. 
(1) If $\delta=0$ (i.e., $L$ has the strong intersection property) then $\mathscr{L}=\{L\}$.

(2) If $\delta=1$ then Ll has 3 members-two incomparable members exceeding $L$.

(3) If $\delta>1$ then $\mathscr{C l}$ has $\max \left\{c, 2^{\circ}\right\}$ members, and the same number of maximal members. If $\delta$ is finite then every maximal chain is finite, and $\delta>c$ if and only if every maximal chain has cardinality at least $c$.

Proof. (1) is obvious, and (2) is an immediate consequence of there being exactly two orders on $Q$, both archimedian. As for (3), Teh [8] has shown that a rational vector space of rank greater than 1 admits at least $c$ orders. If $\delta$ is infinite, there are $2^{\delta}$ orderings of a basis of $V=H_{L} / G$, and each of these yields a distinct lexicographic direct sum ordering of $V$. Thus there are at least $c \cdot 2^{\delta}$ orderings. But since $V$ has $\boldsymbol{\aleph}_{0}, \delta$ elements, there are at most $c \cdot 2^{\delta}$ subsemigroups-let alone that many partial orders - and thus there are exactly $c \cdot 2^{\delta}$ orders on $V$. Each of these yields a distinct maximal member of $O Q(V)$. Now maximal chains in $O Q(V)$ correspond to the collection of all $o$-homomorphic images of a maximal member $[V, P]$ of $O Q(V)$. But $[V, P]$ has at most $c \cdot 2^{\delta}$ convex subgroups and hence admits at most that many 0 -homomorphic images. Thus $|O Q(V)|=$ $c \cdot 2^{\delta}$. If $\delta$ is finite then again from [8], any order on $V$ has finite archimedian rank of at most $\delta$. Using similar arguments, one can show that if $\delta>c$ then the cardinality of the archimedian rank of any order on $V$ is greater than $c$. Thus every maximal chain in $O Q(V)$ also has cardinality greater than $c$. (See [4] for a discourse on archimedian ranks of abelian groups.)

\section{Applications to the study of $\mathscr{L}$.}

LeMmA 4.1. Let $\left(L, \eta_{1}, \eta_{2}\right)$ and $\left(M, \mu_{1}, \mu_{2}\right)$ be amalgamations. The following are equivalent:

(1) $L \succ M$.

(2) For all $h \in H$ and $k \in k, h \eta_{1} \leqq k \eta_{2}$ implies $h \mu_{1} \leqq k \mu_{2}$.

Proof. If $L>M$ via $\theta$ then $h \eta_{1} \leqq k \eta_{2}$ implies

$$
h \mu_{1}=h \eta_{1} \theta \leqq k \eta_{2}=k \mu_{2} \text {. }
$$

Conversely, if (2) is true then the map $\theta: h \eta_{1}+k \eta_{2} \mapsto h \mu_{1}+k \mu_{2}$ is a well-defined $o$-epimorphism by which $L \succ M$.

Let $h \in H$ and $k \in K$. We say that $G$ separates $h$ from $k$ if there exists $g \in G$ such that either $h \leqq g \leqq k$ or $k \leqq g \leqq h$, where at least 
one of the pair of inequalities is strict.

$G$ has a basic archimedian value $\alpha \in A$ if $\alpha$ is the least element of $B$ and of $\Gamma$. Then $G^{\alpha}, H^{\alpha}$, and $K^{\alpha}$ are nonzero archimedian subgroups.

Lemma 4.2. If $G, H$, and $K$ are archimedian, $G \neq 0$, and $G$ does not separate $h$ from $k$, then $h \eta_{1}=k \eta_{2}$ in any archimedian amalgamation $\left(L, \eta_{1}, \eta_{2}\right)$.

Proof. Without loss of generality, $L \subseteq R, \eta_{1}$ and $\eta_{2}$ are inclusion maps, and $1 \in G$. Since $G$ is divisible, $h$ and $k$ determine the same cut of $Q \subseteq G$, whence they must be equal.

THEOREM 4.3. The following are equivalent:

(1) $\mathscr{L}=\mathscr{L}_{0}(G, H, K)$ has one component.

(2) For every $h \in H$ and $k \in K$, either $G$ separates $h$ from $k$, or $G$ has a basic archimedian value $\alpha$ with $h \in H^{\alpha}$ and $k \in K^{\alpha}$.

Proof. Suppose (2) is true, and let $\left(L, \eta_{1}, \eta_{2}\right)$ and $\left(M, \mu_{1}, \mu_{2}\right)$ be minimal. We will show that $L \approx M$. Let $h \eta_{1} \leqq k \eta_{2}$. If $G$ separates $h$ from $k$ then we must have $h \mu_{1} \leqq k \mu_{2}$. If $G$ doesn't separate $h$ from $k$, then the second part of (2) holds. Inspecting the proof of Theorem 1.2, we see that an amalgamation is minimal if and only if it has no proper convex subgroup disjoint from the images of $H$ and $K$. Thus $L$ and $M$ have convex subgroups $F$ and $F^{\prime}$ which cover zero and contain the respective images of $H^{\alpha}$ and $K^{\alpha}$. In fact, $\left(F, \eta_{1}\left|D, \eta_{2}\right| E\right)$ and $\left(F^{\prime}, \mu_{1}\left|D, \mu_{2}\right| E\right)$ are archimedian amalgamations of $G^{\alpha}$ in $H^{\alpha}$ and $K^{\alpha}$. By Lemma 2.3, they are equivalent, and hence $h \mu_{1} \leqq k \mu_{2}$. Thus $L>M$ by Lemma 4.1. But since $L$ is minimal, then $L \approx M$.

Conversely, suppose that (2) fails for some $h \in H$ and $k \in K$, let $h$ have value $\beta \in B$, and let $k$ have value $\gamma \in \Gamma$. We consider two cases, depending on whether or not $\beta$ and $\gamma$ are in $A$. If neither is in $A$ then the hypotheses on $h$ and $k$ imply that for all $\alpha \in A$, either $\alpha$ is greater than both $\beta$ and $\gamma$ or less than both. By the proof of Lemma 2.2 we can find amalgamations $\Delta$ and $\Delta^{\prime}$ of $A$ in $B$ and $\Delta$ such that $\beta<\gamma$ in $\Delta$, but $\gamma<\beta$ in $\Delta^{\prime}$. These then lead to two standard amalgamations, in one of which the image of $h$ is strictly less than that of $k$, and in the other the order is reversed. Thus by Lemma 4.1 they are incomparable. Since standard amalgamations are minimal, $\mathscr{L}$ has at least two components. On the other hand, suppose that $\beta=\gamma=\alpha \in A$, and let $\left(L, \eta_{1}, \eta_{2}\right)$ be any amalgamation. As usual, embed $B$ and $\Gamma$ in $\Delta$, the spine of $L$, and without loss of 
generality consider $L \leqq V(L)$. Let $V=V\left(\Delta^{\prime}, R_{\delta}\right)$ where $\Delta^{\prime}$ arises from $\Delta$ by adjoining $\varepsilon$, and the order is extended by defining $\varepsilon<\delta$ for all $\delta \geqq \alpha$, and $\delta<\varepsilon$ for all $\delta<\alpha$. Let $R_{\delta}=L^{\delta} / L_{\delta}$ and let $R_{\varepsilon}=Q$. $V(L)$ can be naturally embedded in $V$. Since $G \cap Q h=0$, one can find a group homomorphism $\tau: H \rightarrow R_{\varepsilon}$ such that $G+H_{\alpha} \leqq \operatorname{ker}(\tau)$ and $h \tau= \pm 1$, depending on whether $h \eta_{1} \leqq k \eta_{2}$ or $k \eta_{2} \leqq k \eta_{1}$. Finally then, using $\tau$ we can expand to the amalgamation $\left(M, \mu_{1}, \mu_{2}\right)$; i.e., $\mu_{2}=\eta_{2}$, and for $x \in H, x \mu_{1}(\delta)=x \eta_{1}(\delta)$, and $x \mu_{1}(\varepsilon)=x \tau$. By hypothesis $\alpha$ is not the least element of both $B$ and $\Gamma$, so $M$ is also minimal. We claim that $L$ and $M$ are incomparable. $G / G_{\alpha}$, as embedded in $H / H_{\alpha}$ and $K / K_{\alpha}$, does not separate $h+H_{\alpha}$ from $k+K_{\alpha}$. Thus by Lemma 4.2, $h \eta_{1}(\alpha)=k \eta_{2}(\alpha)$. But $\left(h \mu_{1}-k \mu_{2}\right)(\varepsilon)= \pm 1$, the sign chosen so that $h \mu_{1}-k \mu_{2}$ has sign opposite from $h \eta_{1}-k \eta_{2}$. Thus by Lemma 4.1 the amalgamations are incomparable, so again $\mathscr{L}$ has at least two components.

THEOREM 4.4. The following are equivalent:

(1) $|\mathscr{L}|=1$.

(2) For every $h \in H$ and $k \in K, G$ separates $h$ from $k$.

Proof. Suppose (2) is true. By Theorem 4.3, $\mathscr{L}$ has exactly one component with minimal element $L$. But a consequence of the hypothesis is that $L$ has the strong intersection property. Thus by Corollary 3.3, $|\mathscr{L}|=1$. Conversely, if $\mathscr{L}=\left\{\left(L, \eta_{1}, \eta_{2}\right)\right\}$, then in particular condition (2) of Theorem 4.3 holds. But if the second part of that condition is true then by Lemma $4.2 \mathrm{~L}$ fails to have the strong intersection property, whence again by Corollary 3.3 there exists an amalgamation properly exceeding $L$. But this is impossible.

\section{THEOREM 4.5. $\mathscr{L}$ has either one, or at least c, components.}

Proof. Suppose $\mathscr{L}$ has more than one component. By Theorem 4.3 there exist $0<h \in H$ and $0<k \in K$, not having the basic archimedian value if one exists, such that $G$ does not separate $h$ from $k$. Let $h$ have value $\beta$, and $k$ have value $\gamma$. We distinguish two cases. Suppose $\beta$ is the least element of $B$ and $\gamma$ is the least element of $\Gamma$. Since neither is in $A$, there is an amalgamation $\Delta$ of $A$ in $B$ and $\Gamma$ such that $\beta=\gamma$. Since $G_{\beta}=G^{\beta}$, the choice of $\nu_{1_{\beta}}$ : $H^{\beta} / H_{\beta} \rightarrow R_{\beta}$ and $\nu_{2_{\beta}}: K^{\beta} / K_{\beta} \rightarrow R_{\beta}$ in the construction of a standard amalgamation can be arbitrary. We let $\left(h+H_{\beta}\right) \nu_{1_{\beta}}=1$ and for some positive real number $r$ let $\left(k+K_{\beta}\right) \nu_{1_{\beta}}=r$. Evidently the induced standard amalgamations, one for each $r$, are mutually nonequivalent. On the other hand, suppose not both $H_{\beta}$ and $K_{\beta}$ are zero. Let $(L$, $\left.\eta_{1}, \eta_{2}\right)$ be any standard amalgamation. We imitate the construction 
in the latter part of the proof of Theorem 4.3, except that we also choose a group homomorphism $\pi: K \rightarrow R_{\varepsilon}$ such that $G+K_{\beta} \subseteq \operatorname{ker}(\pi)$ and $k \pi=r$. Then we define $\mu_{1}$ as before, and $\mu_{2}$ as $x \mu_{2}(\delta)=x \eta_{2}(\delta)$, and $x \mu_{2}(\varepsilon)=x \pi$. Then the collection of amalgamations, one for each $r$, again form a mutually nonequivalent set of minimal amalgamations.

\section{REFERENCES}

1. B. Banaschewski, Totalgeordnete Moduln, Archiv Math., 7 (1956), 430-440.

2. P. Conrad, Embedding theorems for abelian groups with valuations, Amer. J. Math., 75 (1953), 1-29.

3. L. Fuchs, Partially ordered algebraic systems, Pergammon Press, 1963.

4. D. P. Minassian, Recent developments in the theory of fully ordered groups, Doctoral Dissertation, University of Micigan, 1967.

5. K. R. Pierce, Amalgamations of lattice ordered groups, to appear, Amer. Math. Soc., Transl., 1972.

6. N. R. Reilly, Some applications of wreath products and ultraproducts in the theory of lattice ordered groups, Duke Math. J., 36 (1969), 825-834.

7. E. Szpilrajn, Sur l'extension de l'ordre partiel, Fund. Math., 16 (1930), 386-389.

8. H. H. Teh, Construction of orders in abelian groups, Proc. Cambridge Philos. Soc., 57 (1961), 476-482.

Received August 30, 1971 and in revised form September 11, 1972.

UNIVERSITY OF MISSOURI 



\section{PACIFIC JOURNAL OF MATHEMATICS}

\section{EDITORS}

\author{
H. SAMELSON \\ Stanford University \\ Stanford, California 94305 \\ C. R. HOBBY \\ University of Washington \\ Seattle, Washington 98105
}

\section{J. DugundjI}

Department of Mathematics University of Southern California Los Angeles, California 90007

\section{RICHARD ARENS}

University of California

Los Angeles, California 90024

\section{ASSOCIATE EDITORS}
E. F. BECKENBACH
B. H. NeumanN
F. WOLF
K. YosHIDA

\section{SUPPORTING INSTITUTIONS}

\author{
UNIVERSITY OF BRITISH COLUMBIA \\ CALIFORNIA INSTITUTE OF TECHNOLOGY \\ UNIVERSITY OF CALIFORNIA \\ MONTANA STATE UNIVERSITY \\ UNIVERSITY OF NEVADA \\ NEW MEXICO STATE UNIVERSITY \\ OREGON STATE UNIVERSITY \\ UNIVERSITY OF OREGON \\ OSAKA UNIVERSITY
}

\author{
UNIVERSITY OF SOUTHERN CALIFORNIA \\ STANFORD UNIVERSITY \\ UNIVERSITY OF TOKYO \\ UNIVERSITY OF UTAH \\ WASHINGTON STATE UNIVERSITY \\ UNIVERSITY OF WASHINGTON \\ ${ }^{*} \stackrel{*}{*}{ }^{*}$
AMERICAN MATHEMATICAL SOCIETY
NAVAL WEAPONS CENTER
}

The Supporting Institutions listed above contribute to the cost of publication of this Journal, but they are not owners or publishers and have no responsibility for its content or policies.

Mathematical papers intended for publication in the Pacific Journal of Mathematics should be in typed form or offset-reproduced, (not dittoed), double spaced with large margins. Underline Greek letters in red, German in green, and script in blue. The first paragraph or two must be capable of being used separately as a synopsis of the entire paper. The editorial "we" must not be used in the synopsis, and items of the bibliography should not be cited there unless absolutely necessary, in which case they must be identified by author and Journal, rather than by item number. Manuscripts, in duplicate if possible, may be sent to any one of the four editors. Please classify according to the scheme of Math. Rev. Index to Vol. 39. All other communications to the editors should be addressed to the managing editor, Richard Arens, University of California, Los Angeles, California, 90024.

50 reprints are provided free for each article; additional copies may be obtained at cost in multiples of 50 .

The Pacific Journal of Mathematics is published monthly. Effective with Volume 16 the price per volume (3 numbers) is $\$ 8.00$; single issues, $\$ 3.00$. Special price for current issues to individual faculty members of supporting institutions and to individual members of the American Mathematical Society: $\$ 4.00$ per volume; single issues $\$ 1.50$. Back numbers are available.

Subscriptions, orders for back numbers, and changes of address should be sent to Pacific Journal of Mathematics, 103 Highland Boulevard, Berke'ey, California, 94708.

PUBLISHED BY PACIFIC JOURNAL OF MATHEMATICS, A NON-PROFIT CORPORATION

Printed at Kokusai Bunken Insatsusha (International Academic Printing Co., Ltd.), 270, 3-chome Totsuka-cho, Shinjuku-ku, Tokyo 160, Japan. 


\section{Pacific Journal of Mathematics}

\section{Vol. 43, No. $3 \quad$ May, 1972}

Max K. Agoston, An obstruction to finding a fixed point free map on a manifold.... 543

Nadim A. Assad and William A. Kirk, Fixed point theorems for set-valued mappings

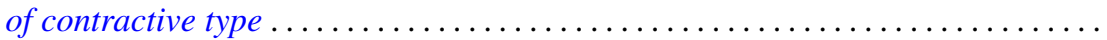

John Winston Bunce, Characterizations of amenable and strongly amenable

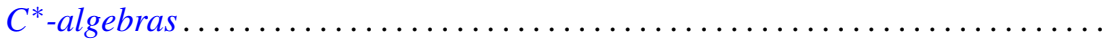

Erik Maurice Ellentuck and Alfred Berry Manaster, The decidability of a class of

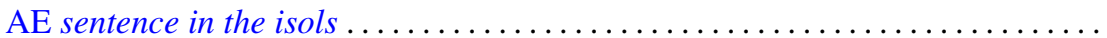

U. Haussmann, The inversion theorem and Plancherel's theorem in a Banach

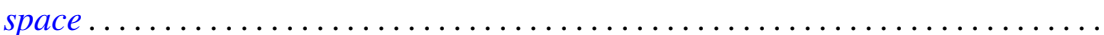

Peter Lawrence Falb and U. Haussmann, Bochner's theorem in infinite dimensions.

Peter Fletcher and William Lindgren, Quasi-uniformities with a transitive base ..... Dennis Garbanati and Robert Charles Thompson, Classes of unimodular abelian

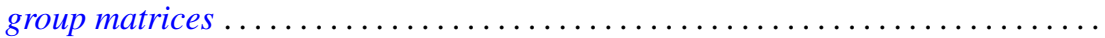

Kenneth Hardy and R. Grant Woods, On c-realcompact spaces and locally bounded normal functions

Manfred Knebusch, Alex I. Rosenberg and Roger P. Ware, Grothendieck and Witt rings of hermitian forms over Dedekind rings .......................

George M. Lewis, Cut loci of points at infinity.

Jerome Irving Malitz and William Nelson Reinhardt, A complete countable $L_{\omega_{1}}^{Q}$ theory with maximal models of many cardinalities . . . . . . . . . . . . . . . . .

Wilfred Dennis Pepe and William P. Ziemer, Slices, multiplicity, and Lebesgue

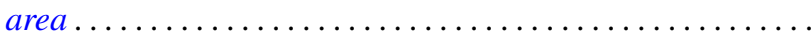

Keith Pierce, Amalgamating abelian ordered groups . .

Stephen James Pride, Residual properties of free groups . . . . . . . . . . . . . 725

Roy Martin Rakestraw, The convex cone of n-monotone functions .

T. Schwartzbauer, Entropy and approximation of measure preserving transformations .

Peter F. Stebe, Invariant functions of an iterative process for maximization of a polynomial...

Kondagunta Sundaresan and Wojbor Woyczynski, L-orthogonally scattered

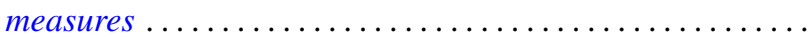

Kyle David Wallace, $C_{\lambda}$-groups and $\lambda$-basic subgroups $\ldots \ldots \ldots$

Barnet Mordecai Weinstock, Approximation by holomorphic functions on certain

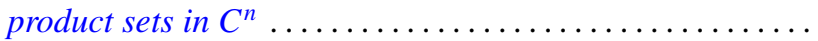

Donald Steven Passman, Corrections to: "Isomorphic groups and group rings”.

Don David Porter, Correction to: "Symplectic bordism, Stiefel-Whitney numbers, and a Novikov resolution"

John Ben Butler, Jr., Correction to: “Almost smooth perturbations of self-adjoint operators".

Constantine G. Lascarides, Correction to: "A study of certain sequence spaces of Maddox and a generalization of a theorem of Iyer" ...... ...

George A. Elliott, Correction to: "An extension of some results of takesaki in the reduction theory of von neumann algebras" ......................... 